\title{
A Remark on the Micro-local Resolvent Estimates for Two Body Schrödinger Operators
}

\author{
By \\ Hiroshi IsOzAKI* and Hitoshi KITADA**
}

\section{Introduction}

In this paper, we shall deal with the estimates for the resolvent of a Schrödinger operator multiplied by some pseudo-differential operators.

We consider the Schrödinger operator $H=-\Delta+V(x)$ in $L^{2}\left(\boldsymbol{R}^{n}\right)(n \geqq 2)$, where $\Delta$ denotes the Laplacian in $\boldsymbol{R}^{n}$. We assume that $V(x)$ is a real-valied $C^{\infty}$-function on $\boldsymbol{R}^{n}$ and for a constant $0<\varepsilon_{0}<1$

$$
\partial_{x}^{a} V(x)=O\left(|x|^{-|a|-\varepsilon_{0}}\right) \quad \text { as } \quad|x| \rightarrow \infty,
$$

for all multi-index $\alpha$, where $\partial_{x}^{\alpha}=\left(\partial / \partial x_{1}\right)^{a_{1}} \cdots\left(\partial / \partial x_{n}\right)^{a}$ and $|\alpha|=\alpha_{1}+\cdots+\alpha_{n}$. One can also allow certain local singularities for $V$, which, however, is omitted here for the sake of simplicity. Let $R(z)=(H-z)^{-1}$ for $\operatorname{Im} z \neq 0$ and $\langle x\rangle=$ $\left(1+|x|^{2}\right)^{1 / 2}$ for $x \in \boldsymbol{R}^{n}$. Let $\|\cdot\|$ denote the operator norm in $L^{2}\left(\boldsymbol{R}^{n}\right)$. Then, as is well-known,

$$
\left\|\langle x\rangle^{-s} R(\lambda \pm i 0)\langle x\rangle^{-s}\right\| \leqq C / \sqrt{\lambda},
$$

for any $s>1 / 2$ and $\lambda>a_{0}, a_{0}$ being an arbitrarily fixed positive constant (see e.g. [1], Theorem 1.2). Our goal is to improve the estimate (0.2) by multiplying some pseudo-differential operators (Ps. D. Op.'s).

Let us consider the Ps. D. Op.'s $P_{ \pm}$with symbols $p_{ \pm}(x, \xi)$ having the following properties:

(0.4) for a constant $\varepsilon>0, \quad p_{ \pm}(x, \xi)=0$ if $|x|<\varepsilon$ or $|\xi|<\varepsilon$,

(0.5) there exist constants $-1<\mu_{ \pm}<1$ such that

$$
p_{+}(x, \xi)=0 \text { if } \hat{x} \cdot \hat{\xi}<\mu_{+},
$$

Communicated by S. Matsuura, January 19, 1984.

* Department of Mathematics, Kyoto University, Kyoto 606, Japan.

** Department of Mathematics, College of General Education, University of Tokyo, Komaba. Meguro-ku, Tokyo 153, Japan. 


$$
p_{-}(x, \xi)=0 \quad \text { if } \hat{x} \cdot \hat{\xi}>\mu_{-}, \quad(\hat{x}=x /|x|, \hat{\xi}=\xi /|\xi|) .
$$

Our results of this paper are summarized in the following theorems.

Theorem 1. Let $P_{ \pm}$be as above. Then for any $s \geqq 0$ and $\delta>1$

$$
\left\|\langle x\rangle^{s} P_{\mp} R(\lambda \pm i 0)\langle x\rangle^{-(s+\delta)}\right\| \leqq C / \sqrt{\lambda},
$$

where the constant $C$ is independent of $\lambda>a_{0}>0, a_{0}$ being an arbitrarily fixed constant.

Theorem 2. Let $P_{ \pm}$be as above. If $\mu_{+}>\mu_{-}$, we have for any $s>0$

$$
\left\|\langle x\rangle^{s} P_{\mp} R(\lambda \pm i 0) P_{ \pm}\langle x\rangle^{s}\right\| \leqq C / \sqrt{\lambda},
$$

where the constant $C$ is independent of $\lambda>a_{0}>0$.

Choose a $C^{\infty}$-function $\chi(x)$ such that $\chi(x)=1$ for $|x|<1, \chi(x)=0$ for $|x|>2$ and set $V_{\jmath}(x)=\chi(x / j) V(x)$. Let $H_{\jmath}=-\Delta+V_{\jmath}$ and $R_{\jmath}(z)=\left(H_{\jmath}-z\right)^{-1}$. Then we have

Theorem 3. Let $P_{ \pm}$be as above. If $\mu_{+}>\mu_{-}$, we have for any $s>0$

$$
\left\|\langle x\rangle^{s} P_{-}\left(R(\lambda+i 0)-R_{j}(\lambda+i 0)\right) P_{+}\langle x\rangle^{s}\right\| \leqq C j^{-\varepsilon_{0}} / \lambda,
$$

where $C$ is a constant independent of $j$ and $\lambda>a_{0}>0$.

These theorems have not only their own interests but also important applications to the study of $S$-matrices for Schrödinger operators.

We have already discussed similar theorems in [1] by the stationary method. We localized the Schrödinger equation in the momentum space and reduced it to a Hilbert space valued ordinary differential equation with Ps. D. Op. coefficients, which we learned from Agmon's lecture at Kyoto University in 1977. In this paper, we propose to discuss the same problem by the time-dependent method. That is, we construct a parametrix for the unitary group $e^{-i t H}$ and reduce the estimates for the resolvent to those of the parametrix. For the unperturbed operator $H_{0}=-\Delta$, this method clarifies the close connection between the resolvent estimates and the propagation properties of the scattering states.

In Section 1, we explain the idea of the proof for $H_{0}=-\Delta$. We prepare in Section 2 a parametrix for $e^{-i t H}$ and obtain its estimates. Theorems $1,2,3$ will be proved in Sections 3, 4 and 5, respectively. In Sections 6, 7, some technical results for pseudo-differential operators (Ps. D. Op.'s) and Fourier integral operators (F.I.Op.'s) are proved.

The notations we used here are almost standard. $\mathscr{B}\left(\boldsymbol{R}^{n}\right)$ denotes the space of smooth functions on $\boldsymbol{R}^{n}$ with bounded derivatives. $C_{0}^{\infty}\left(\boldsymbol{R}^{n}\right)$ is the totality of smooth functions with compact support. For $x \in \boldsymbol{R}^{n},\langle x\rangle=\left(1+|x|^{2}\right)^{1 / 2}$ and $\hat{x}=$ $x /|x| . \quad D_{x}^{\alpha}=(-i)^{|\alpha|} \partial_{x}^{\alpha} . \quad \hat{f}(\xi)$ is the Fourier transform of $f$ : 


$$
\hat{f}(\xi)=\int_{\boldsymbol{R}^{n}} e^{-i x \xi} f(x) d x,
$$

$d \xi=(2 \pi)^{-n} d \xi, d \xi$ being the Lebesgue measure on $\boldsymbol{R}^{n}$.

Finally, the authors would like to express their deep gratitude to Professor J. Uchiyama for his kind advices and various instructions for this paper.

\section{$\S 1$. Free Time Evolutions and Resolvent Estimates}

As has been stated in the introduction, our micro-local resolvent estimates are closely related with the propagation properties of scattering states. Let us explain it in this section taking $H_{0}=-\Delta$ as an example, although it is not logically necessary.

Let $R_{0}(z)=\left(H_{0}-z\right)^{-1}$. Let $P_{ \pm}$be the Ps. D. Op.'s with symbols $p_{ \pm}(x, \xi)$ satisfying (0.3)-(0.5). We assume for the sake of simplicity that

$$
\operatorname{supp}_{\xi} p_{ \pm}(x, \xi) \subset\{\xi ; a<|\xi|<b\} \quad(0<a<b<\infty) .
$$

Then we have for $f \in C_{0}^{\infty}\left(\boldsymbol{R}^{n}\right)$ and $r, s \geqq 0$

$$
\langle x\rangle^{-r} e^{-i t H_{0}} P_{+}^{*}\langle x\rangle^{s} f(x)=\iint e^{\imath\left[(x-y) \xi-t|\xi|^{2}\right]}\langle x\rangle^{-r} \overline{p_{+}(y, \xi)}\langle y\rangle^{s} f(y) d y d \xi .
$$

For the moment, we assume that $r$ and $s$ are non-negative integers such that $r \geqq s$. On the support of $p_{+}(y, \xi)$, we have $\hat{y} \cdot \hat{\xi} \geqq \mu_{+}>-1$. Therefore for $t \geqq 0$, $|y+2 \xi t| \geqq C(|y|+t|\xi|)$, for a constant $C>0$. Let $S(\xi, y ; t)=y \xi+t|\xi|^{2}$. Then

$$
\left|\nabla_{\xi} S(\xi, y ; t)\right| \geqq C(|y|+t|\xi|) .
$$

Making use of the relation

$$
e^{-i S}=i\left|\nabla_{\xi} S\right|^{-2} \nabla_{\xi} S \cdot \nabla_{\xi} e^{-\imath S}
$$

we have by integrating by parts $r$ times

$$
\langle x\rangle^{-r} e^{-i t H_{0}} P_{+}^{*}\langle x\rangle^{s} f(x)=\sum_{m}^{\text {finite }} \iint e^{\imath\left[(x-y) \xi-t|\xi|{ }^{2}\right]} a_{m}(x) b_{m}(y, \xi ; t) f(y) d y d \xi
$$

where $a_{m}(x) \in \mathscr{B}\left(\mathbb{R}^{n}\right), b_{m}(y, \xi ; t)$ is a $C^{\infty}$-function such that

$$
\left|D_{y}^{\alpha} D_{\xi}^{\beta} b_{m}(y, \xi ; t)\right| \leqq C_{\alpha \beta}(1+|y|+t|\xi|)^{-(r-s)} \quad(t \geqq 0) .
$$

Let $A_{m}$ and $B_{m}(t)$ be the Ps. D. Op.'s with symbols $a_{m}(x)$ and $\overline{b_{m}(x, \xi ; t)}$, respectively. Then one can rewrite the above equality as follows:

$$
\langle x\rangle^{-r} e^{-i L H_{0}} P_{+}^{*}\langle x\rangle^{s}=\sum_{m} A_{m} e^{-i \underline{H}{ }_{0}} D_{m}(t)^{*} .
$$

From (1.2), one can easily see that $\left\|B_{m}(t)\right\| \leqq C(1+t)^{-(r-s)}$ for $t \geqq 0$. Thus we have for $t \geqq 0$

$$
\left\|\langle x\rangle^{-r} e^{-i t H_{0}} P_{+}^{*}\langle x\rangle^{s}\right\| \leqq C(1+t)^{-(r-s)} .
$$


By an interpolation, the above estimates easily extends to any $r \geqq s \geqq 0$. To prove (1.4) for $t \leqq 0$, we have only to replace $P_{+}$by $P_{-}$. We have thus shown the following

Lemma 1.1. For any $\delta, s \geqq 0$, there exists a constant $C>0$ such that

$$
\begin{array}{ll}
\left\|\langle x\rangle^{-(s+\delta)} e^{-i t H_{0}} P_{+}^{*}\langle x\rangle^{s}\right\| \leqq C(1+t)^{-\delta} & (t \geqq 0), \\
\left\|\langle x\rangle^{-(s+\delta)} e^{-i t H_{0}} P^{*}\langle x\rangle^{s}\right\| \leqq C(1-t)^{-\delta} & (t \leqq 0) .
\end{array}
$$

We now make use of the following formulae

$$
\begin{array}{ll}
R_{0}(z)=i \int_{0}^{\infty} e^{-i t\left(H_{0}-z\right)} d t \quad(\operatorname{Im} z>0), \\
R_{0}(z)=-i \int_{-\infty}^{0} e^{-i t\left(H_{0}-z\right)} d t \quad(\operatorname{Im} z<0),
\end{array}
$$

to see

Theorem 1.2. For $s \geqq 0$ and $\delta>1$,

$$
\begin{aligned}
& \left\|\langle x\rangle^{-(s+\delta)} R_{0}(\lambda \pm i 0) P_{ \pm}^{*}\langle x\rangle^{s}\right\| \leqq C, \\
& \left\|\langle x\rangle^{s} P_{\mp} R_{0}(\lambda \pm i 0)\langle x\rangle^{-(s+\delta)}\right\| \leqq C,
\end{aligned}
$$

where the constant $C$ is independent of $\lambda>0$.

Next let us consider

$$
\langle x\rangle^{s} P_{-} e^{-i t H_{0}} P_{+}^{*}\langle x\rangle^{s} f(x)=\iint e^{i\left[(x-y) \xi-\left.t|\xi|\right|^{2}\right.}\langle x\rangle^{s} p_{-}(x, \xi) \overline{p_{+}(y, \xi)}\langle y\rangle^{s} f(y) d y d \xi,
$$

For $\xi \neq 0$, define two cones $\Gamma_{ \pm}$by

$$
\Gamma_{+}=\left\{y ; \hat{y} \cdot \hat{\xi} \geqq \mu_{+}\right\}, \quad \Gamma_{-}=\left\{x ; \hat{x} \cdot \hat{\xi} \leqq \mu_{-}\right\} .
$$

On the support of $p_{-}(x, \xi) \overline{p_{+}(y, \xi)}, x \in \Gamma_{-}$and $y+2 t \xi \in \Gamma_{+}$for $t \geqq 0$. Therefore if $\mu_{+}>\mu_{-}$, a simple trigonometry shows that

$$
\begin{aligned}
|x-(y+2 t \xi)| & \geqq C(|x|+|y+2 t \xi|) \\
& \geqq C(|x|+|y|+t|\xi|) \quad(t \geqq 0),
\end{aligned}
$$

with a constant $C>0$. Let $S(x, \xi, y ; t)=(x-y) \xi-t|\xi|^{2}$. Then on the support of $p_{-}(x, \xi) \overline{p_{+}(y, \xi)}$

$$
\left|\Gamma_{\xi} S(x, \xi, y ; t)\right| \geqq C(|x|+|y|+t|\xi|) .
$$

We now integrate by parts $N$ times using the relation $e^{i S}=-i\left|\nabla_{\xi} S\right|^{-2} \nabla_{\xi} S \cdot \nabla_{\xi} e^{i S}$ to obtain

$$
\langle x\rangle^{s} P_{-} e^{-i t H_{0}} P^{*}\langle x\rangle^{s} f(x)=\iint e^{i S(x, \xi, y ; t)} a_{N}(x, \xi, y ; t) f(y) d y d \xi,
$$


where

$$
\left|D_{x}^{a} D_{\xi}^{\beta} D_{y}^{\gamma} a_{N}(x, \xi, y ; t)\right| \leqq C(1+|x|+|y|+t|\xi|)^{-(N-2 s)} \quad(t \geqq 0) .
$$

$\langle x\rangle^{s} P_{-} e^{-i t I I} P_{+}^{*}\langle x\rangle^{s}$ can be regarded as a Ps.D.Op. with symbol $e^{-\left.\left.i t\right|_{5}\right|^{2}} a_{N}(x, \xi, y ; t)$. Thus taking $N$ large enough, we have

Lemma 1.3. If $\mu_{+}>\mu_{-}$, we have for any $N, s \geqq 0$,

$$
\left\|\langle x\rangle^{s} P_{-} e^{-i t H_{0}} P_{+}^{*}\langle x\rangle^{s}\right\| \leqq C(1+t)^{-N} \quad(t \geqq 0) .
$$

Passing to the Laplace transform, we obtain

Theorem 1.4. If $\mu_{+}>\mu_{-}$, we have for any $s>0$

$$
\left\|\langle x\rangle^{s} P_{-} R_{0}(\lambda+i 0) P_{+}^{*}\langle x\rangle^{s}\right\| \leqq C,
$$

for a constant $C>0$ independent of $\lambda>0$.

The above Theorems 1.2 and 1.4 are particular cases of our results and suggest how the propagation properties of $e^{-\imath t H_{0}}$ affect to the resolvent estimates. In order to generalize the above results to the perturbed operator $H$, we have to introduce a parametrix at infinity for $e^{-i t H}$.

\section{§2. A Parametrix at Infinity for $e^{-i t H}$}

First we take note of the fact that one can obtain the same estimates as in Section 1 using suitable F.I.Op.'s instead of Ps. D. Op.'s. More precisely, we consider F.I.Op.'s $A, B_{ \pm}(\lambda)$ defined by

$$
\begin{aligned}
& A f(x)=\int e^{i \dot{\varphi}(x, \xi)} a(x, \xi) \hat{f}(\xi) d \xi, \\
& B_{ \pm}(\lambda) f(x)=\int e^{i \dot{(\dot{ }(x, \xi)}} b_{ \pm}(x, \xi ; \lambda) \hat{f}(\xi) d \xi .
\end{aligned}
$$

Assume that the phase function $\dot{\phi}(x, \xi)$ is real-valued and smooth on $\mathbb{R}^{n} \times \mathbb{R}^{n}$ and that

$$
\left|D_{x}^{\alpha} D_{\xi}^{\beta}(\phi(x, \xi)-x \cdot \xi)\right| \leqq C_{a \beta}\langle x\rangle^{1-s-|\alpha|}
$$

for some $\varepsilon>0$ and all $\alpha, \beta$, and

$$
\sup _{x, \xi}\left|\left(\frac{\partial^{2}}{\partial x_{i} \partial \xi_{j}} \phi(x, \xi)\right)-I\right|<1 / 2,
$$

where $I$ is the $n \times n$ identity matrix.

As for the amplitude functions $a(x, \xi), b_{x}(x, \xi ; \lambda)$, we assume that

$$
\begin{aligned}
& a(x, \xi) \in \mathscr{B}\left(\mathbb{R}^{2 n}\right), \\
& \left|D_{x}^{\alpha} D_{\xi}^{3} b_{ \pm}(x, \xi ; \lambda)\right| \leqq C_{a, 3}\langle x\rangle^{-|x|},
\end{aligned}
$$


for any $\lambda>a_{0}>0$, where $a_{0}$ is an arbitrarily fixed constant,

(2.9) there exist constants $-1<\mu_{ \pm}<1$ such that

$$
\begin{array}{lll}
b_{+}(x, \xi ; \lambda)=0 & \text { if } & \hat{x} \cdot \hat{\xi}<\mu_{+}, \\
b_{-}(x, \xi ; \lambda)=0 & \text { if } \quad \hat{x} \cdot \hat{\xi}>\mu_{-} .
\end{array}
$$

Lemma 2.1. $A, B_{ \pm}(\lambda)$, defined above, are $L^{2}$-bounded. Moreover we have:

(1) For any $s, \delta \geqq 0$,

$$
\left\|\langle x\rangle^{-(s+\delta)} A e^{-i t H_{0}} B_{ \pm}(\lambda) *\langle x\rangle^{s}\right\| \leqq C(1+t \sqrt{\lambda})^{-\delta}
$$

for any $\lambda>a_{0}$ and $\pm t \geqq 0$.

(2) Suppose there exists a constant $\mu$ such that $-1<\mu<\mu_{+}<1$ and $a(x, \xi)=0$ if $\hat{x} \cdot \hat{\xi}>\mu$. Then for any $s, N>0$

$$
\left\|\langle x\rangle^{s} A e^{-i t H_{0}} B_{+}(\lambda)^{*}\langle x\rangle^{s}\right\| \leqq C(1+t \sqrt{\lambda})^{-N}
$$

for $\lambda>a_{0}$ and $t \geqq 0$.

The above lemma is proved by integration by parts in essentially the same methods as given in Section 1. See [2], Lemma 3.3 for details.

In [2], we introduced a parametrix for $e^{-i t H}$ in the form of a F.I.Op. which gives an approximate behavior of $e^{-i t H}$ in an appropriate region of the phase space and proved the asymptotic completeness of modified wave operators for $H$. In the following, we show that its Laplace transform is a nice parametrix for the resolvent $R(z)=(H-z)^{-1}$. Our method relies on that of geometrical optics. We begin with the choice of a phase function.

Theorem 2.2. Let $\varepsilon>0$ be a sufficiently small constant. Choose $d>0$ arbitrarily. Then there exists a real function $\phi(x, \xi) \in C^{\infty}\left(\boldsymbol{R}^{n} \times \boldsymbol{R}^{n}\right)$ having the following properties:

(1) There exists a constant $R>0$ such that for $|x|>R,|\xi|>d / 2$ and $\hat{x} \cdot \hat{\xi}>$ $-1+\varepsilon / 2, \phi(x, \xi)$ solves the eikonal equation

$$
\left|\nabla_{x} \phi(x, \xi)\right|^{2}+V(x)=|\xi|^{2} .
$$

(2) For any multi-indices $\alpha, \beta$

$$
\left|D_{x}^{\alpha} D_{\xi}^{\beta}(\phi(x, \xi)-x \cdot \xi)\right| \leqq C_{a \beta}\langle x\rangle^{1-\varepsilon_{0}-|\alpha|}\langle\xi\rangle^{-1},
$$

for any $x, \xi \in \mathbb{R}^{n}$, where $\varepsilon_{0}$ is the constant given in (0.1).

(3) $\sup _{x, \xi}\left|\left(\frac{\partial^{2}}{\partial x_{i} \partial \xi} \phi(x, \xi)\right)-I\right|<1 / 2$. 
For the proof, see [2], Theorem 2.5.

Next we turn to the transport equation. Let us take note of the following identity

$$
\begin{aligned}
e^{-\imath \dot{\phi}}\left(-\Delta+V-|\xi|^{2}\right) e^{\imath \dot{\varphi}} a= & \left(|\nabla \phi|^{2}+V-|\xi|^{2}\right) a \\
& -2 i \nabla \phi \cdot \nabla a-i(\Delta \phi) a-\Delta a .
\end{aligned}
$$

We set $a=\sum_{m=0}^{N} a_{m}$, where $a_{0}=1$. Then the right-hand side of (2.10) takes the following form

$$
\begin{aligned}
& \left(|\nabla \phi|^{2}+V-|\xi|^{2}\right) a-i(\Delta \phi) a_{0} \\
& \quad-2 i \nabla \phi \cdot \nabla a_{1}-i(\Delta \phi) a_{1}-\Delta a_{1} \\
& \quad \cdots \quad \ldots \\
& \quad-2 i \nabla \phi \cdot \nabla a_{N}-i(\Delta \phi) a_{N}-\Delta a_{N} .
\end{aligned}
$$

Define a region $\Omega=\{(x, \xi) ;|\xi|>d / 2,|x|>R, \hat{x} \cdot \hat{\xi}>-1+\varepsilon / 2\}$, where $\varepsilon, d, R$ are the constants specified in Theorem $2.2(1)$. We construct $a_{m}(x, \xi)$ in such a way that

$$
2 i \nabla \phi \cdot \nabla a_{m}+i(\Delta \phi) a_{m-1}+\Delta a_{m-1}=0, \quad m=1,2, \cdots,
$$

for $(x, \xi) \in \Omega$. Then for $(x, \xi) \in \Omega$

$$
e^{-i \dot{\varphi}}\left(-\Delta+V-|\xi|^{2}\right) e^{\imath \dot{\varphi}} \sum_{m=0}^{N} a_{m}=-i(\Delta \phi) a_{N}-\Delta a_{N} \text {. }
$$

The equation (2.11) is a first order partial differential equation and can be solved by the method of characteristics. From Theorem 2.2, it follows that

$$
\nabla \phi(x, \xi)=\xi+O\left(|x|^{-\varepsilon_{0}}\right), \quad \Delta \phi(x, \xi)=O\left(|x|^{-1-\varepsilon_{0}}\right)
$$

as $|x| \rightarrow \infty,(x, \xi) \in \Omega$. Thus one can integrate (2.11) from the infinity of $\xi$ direction. Furthermore one can show inductively that

$$
\left|D_{x}^{\alpha} D_{\xi}^{\beta} a_{m}(x, \xi)\right| \leqq C_{\alpha \beta m}\langle\xi\rangle^{-1}\langle x\rangle^{-|a|-m \varepsilon_{0}}
$$

for $(x, \xi) \in \Omega, m \geqq 1$. We omit the proof of these facts in order not to make this paper bulky, partly because it is almost routine. It would be worthwhile, however, to note the short range case:

$$
D_{x}^{\alpha} V(x)=O\left(|x|^{-|a|-1-\delta}\right) \quad(\delta>0),
$$

since in this case everything can be written down explicitly. In the case of the short-range potential, we should take $x \cdot \xi$ as $\phi(x, \xi)$. Then (2.10) can be written as follows:

$$
e^{-i x \xi}\left(-\Delta+V-|\xi|^{2}\right) e^{2 x \xi} a=V a-2 i \xi \cdot \nabla a-\Delta a .
$$

We set as above $a=\sum_{m=0}^{N} a_{m}, a_{0}=1$. Then the right-hand side takes the following form 


$$
\begin{aligned}
& -2 i \xi \cdot \nabla a_{0}-\Delta a_{0}+V a_{0} \\
& -2 i \xi \cdot \nabla a_{1}-\Delta a_{1}+V a_{1} \\
& \ldots \quad \ldots \\
& -2 i \xi \cdot \nabla a_{N}-\Delta a_{N}+V a_{N} .
\end{aligned}
$$

Thus we have only to solve

$$
2 i \xi \cdot \nabla a_{m}=V a_{m-1}-\Delta a_{m-1},
$$

which can be easily integrated from infinity:

$$
a_{m}(x, \xi)=\frac{i}{2|\xi|} \int_{0}^{\infty}\left\{V(x+\hat{\xi} t) a_{m-1}(x+\hat{\xi} t, \xi)-\left(\Delta a_{m-1}\right)(x+\hat{\xi} t, \xi)\right\} d t .
$$

It is now easy to show by induction that $a_{m}(x, \xi)$ satisfies

$$
\begin{array}{ll}
\left|D_{x}^{\alpha} D_{\xi}^{3} a_{m}(x, \xi)\right| \leqq C_{\alpha \beta m}\langle\xi\rangle^{-m-|\beta|}\langle x\rangle^{-|a|-m \delta} \quad \text { if } & 0<\delta<1, \\
\left|D_{x}^{\alpha} D_{\xi}^{\beta} a_{m}(x, \xi)\right| \leqq C_{\alpha \beta m}\langle\xi\rangle^{-m-|\beta|}\langle x\rangle^{-|\alpha|-(m-1)-\delta} & \text { if } \quad \delta>1,
\end{array}
$$

for $m \geqq 1,|\xi|>d / 2, \hat{x} \cdot \hat{\xi}>-1+\varepsilon / 2$.

We now use the well-known technique of constructing a $C^{\infty}$-function with given asymptotic expansions (see, e.g. Kumanogo [4], p. 77). Choose $\chi(x) \in C^{\infty}\left(\boldsymbol{R}^{n}\right)$ such that $\chi(x)=1$ for $|x|>2, \chi(x)=0$ for $|x|<1$. For a suitable choice of a sequence $R_{0}<R_{1}<\cdots \rightarrow \infty \quad\left(R_{0}=R, R\right.$ being the constant specified in Theorem 2.2 (1)), the function $\tilde{a}(x, \xi)$ defined by

$$
\tilde{a}(x, \xi)=\sum_{m=0}^{\infty} \chi\left(x / R_{m}\right) a_{m}(x, \xi)
$$

is convergent and smooth on $\Omega$, and for $M \geqq 1$,

$$
\left|D_{x}^{\alpha} D_{\xi}^{\beta}\left(\sum_{m=M}^{\infty} \chi\left(x / R_{m}\right) a_{m}(x, \xi)\right)\right| \leqq C_{\alpha \beta M}\langle x\rangle^{-|\alpha|-. M \varepsilon_{0}}\langle\xi\rangle^{-1}
$$

for $(x, \xi) \in \Omega$. Moreover, if we let

$$
\tilde{G}(x, \xi)=e^{-i \dot{\varphi}^{\prime}(x, \xi)}\left(-\Delta+V(x)-|\xi|^{2}\right)\left(e^{i \rho(x, \xi)} \tilde{a}(x, \xi)\right),
$$

it satisfies for any $N$ and $(x, \xi) \in \Omega$,

$$
\left|D_{x}^{a} D_{\xi}^{\beta} \tilde{G}(x, \xi)\right| \leqq C_{\alpha \beta N}\langle x\rangle^{-N}\langle\xi\rangle .
$$

Finally we choose $\rho_{0}(t), \rho_{1}(t) \in C^{\infty}\left(\boldsymbol{R}^{1}\right)$ such that $\rho_{0}(t)=1$ for $t>d, \rho_{0}(t)=0$ for $t<d / 2$ and $\rho_{1}(t)=1$ for $t>-1+\varepsilon, \rho_{1}(t)=0$ for $t<-1+\varepsilon / 2$, and let

$$
a(x, \xi)=\tilde{a}(x, \xi) \rho_{0}(|\xi|) \rho_{1}(\hat{x} \cdot \hat{\xi}) .
$$

Then one can easily show the following

Theorem 2.3. $a(x, \xi)$, constructed above, has the following properties:

$$
\left|D_{x}^{\alpha} D_{\xi}^{\beta}(a(x, \xi)-1)\right| \leqq C_{a \beta}\langle\xi\rangle^{-1}\langle x\rangle^{-|\alpha|-\varepsilon_{0}}
$$


if $|\xi|>d, \hat{x} \cdot \hat{\xi}>-1+\varepsilon,|x|>2 R, R$ being the constant specified in Theorem 2.2 (1). $a(x, \xi)=0$ if $|\xi|<d / 2$ or $\hat{x} \cdot \hat{\xi}<-1+\varepsilon / 2$ or $|x|<R$.

(2) Let $G(x, \xi)=e^{-\imath \dot{\xi}^{(x, \xi)}}\left(-\Delta+V(x)-|\xi|^{2}\right)\left(e^{\imath(\rho)(x, \xi)} a(x, \xi)\right)$. Then for $\hat{x} \cdot \hat{\xi}>$ $-1+\varepsilon$, we have for any $N>0$

If $\hat{x} \cdot \hat{\xi}<-1+\varepsilon$,

$$
\left|D_{x}^{a} D_{\xi}^{\beta} G(x, \xi)\right| \leqq C_{a, N}\langle\xi\rangle\langle x\rangle^{-N} .
$$

$$
\left|D_{x}^{a} D_{\xi}^{\beta} G(x, \xi)\right| \leqq C_{a, 3}\langle\xi\rangle\langle x\rangle^{-1-|a|} \text {. }
$$

We are now in a position to construct a parametrix for $e^{-i t H}$.

Definition 2.4. Let $\phi(x, \xi), a(x, \xi)$ and $G(x, \xi)$ be as in Theorems 2.2 and 2.3 , respectively. Let $b_{ \pm}(x, \xi ; \lambda)$ satisfy $(2.6)-(2.9)$. We define :

$$
\begin{aligned}
& A_{+} f(x)=\int e^{i \dot{\xi}(x, \xi)} a(x, \xi) \hat{f}(\xi) d \xi, \\
& A_{-} f(x)=\int e^{-\imath \dot{\varphi}(x,-\xi)} \overline{a(x,-\xi)} \hat{f}(\xi) d \xi, \\
& B_{ \pm}(\lambda) f(x)=\int e^{ \pm i \xi(x, \pm \xi)} b_{ \pm}(x, \xi ; \lambda) \hat{f}(\xi) d \xi, \\
& G_{+} f(x)=\int e^{\imath \dot{\xi}(x, \hat{\xi})} G(x, \xi) \hat{f}(\xi) d \xi, \\
& G_{-} f(x)=\int e^{-\imath \dot{\xi}(x,-\xi)} \overline{G(x,-\xi) \hat{f}(\xi) d \xi,} \\
& U_{ \pm}(t ; \lambda)=A_{ \pm} e^{-i t H_{0}} B_{ \pm}(\lambda) *, \\
& G_{ \pm}(t ; \lambda)=G_{ \pm} e^{-i t H_{0}} B_{ \pm}(\lambda) * .
\end{aligned}
$$

One should take notice of the following fact. Let $S(x, \xi, y ; t)=\phi(x, \xi)-$ $\phi(y, \xi)-t|\xi|^{2}$. Then

$$
\begin{aligned}
& U_{+}(t ; \lambda) f(x)=\iint e^{i S(x, \xi, y ; t)} a(x, \xi) \overline{b_{+}(y, \xi ; \lambda)} f(y) d y d \xi, \\
& G_{+}(t ; \lambda) f(x)=\iint e^{i S(x, \xi, y ; t)} G(x, \xi) \overline{b_{+}(y, \xi ; \lambda)} f(y) d y d \xi .
\end{aligned}
$$

We also have

$$
\begin{aligned}
& \overline{U_{-}(-t ; \lambda) f(x)}=\iint e^{\imath S(x, \xi, y ; t)} a(x, \xi) b_{-}(y,-\xi ; \lambda) \overline{f(y)} d y d \xi, \\
& \overline{G_{-}(-t ; \lambda) f(x)}=\iint e^{i S(x, \xi, y ; t)} G(x, \xi) b_{-}(y,-\xi ; \lambda) \overline{f(y)} d y d \xi .
\end{aligned}
$$

Thus the estimates for $U_{+}(t ; \lambda), G_{+}(t ; \lambda)$ for $t \geqq 0$ are easily translated into those for $U_{-}(t ; \lambda), G_{-}(t ; \lambda)$ for $t \leqq 0$.

A straightforward calculation shows that 


$$
i \frac{\partial}{\partial t} U_{ \pm}(t ; \lambda)=H U_{ \pm}(t ; \lambda)-G_{ \pm}(t ; \lambda) .
$$

Therefore, since $U_{ \pm}(0 ; \lambda)=A_{ \pm} B_{ \pm}(\lambda) *$,

$$
e^{-i t H} A_{ \pm} B_{ \pm}(\lambda) *=U_{ \pm}(t ; \lambda)-i \int_{0}^{t} e^{-\imath(t-s) H} G_{ \pm}(s ; \lambda) d s .
$$

Lemma 2.5. (1) For any $s, \delta \geqq 0$, we have

$$
\left\|\langle x\rangle^{-(s+\delta)} U_{+}(t ; \lambda)\langle x\rangle^{s}\right\| \leqq C(1+t \sqrt{\lambda})^{-\delta}
$$

for $\lambda>a_{0}>0$ and $t \geqq 0$.

(2) Let $\mu_{+}$and $\varepsilon$ be as in (2.9) and Theorem 2.3 (2). If $\mu_{+}>-1+\varepsilon$, we have for any $s, N \geqq 0$

$$
\left\|\langle x\rangle^{s}\left\langle D_{x}\right\rangle^{-1} G_{+}(t ; \lambda)\langle x\rangle^{s}\right\| \leqq C(1+t \sqrt{\lambda})^{-N},
$$

for $\lambda>a_{0}>0, t \geqq 0$, where $\left\langle D_{x}\right\rangle^{-1}$ is a Ps. D. Op. with symbol $\langle\xi\rangle^{-1}$.

Proof. (1) is a direct consequence of Lemma 2.1 (1). We prove (2). By Theorem 6.2 in Section 6, for large $! N>0,\left\langle D_{x}\right\rangle^{-1} G_{+}=A+P_{N}$, where $P_{N}$ is a Ps. D. Op. with symbol $p_{N}(x, \xi)$ such that

$$
\left|D_{x}^{\alpha} D_{\xi}^{\beta} p_{N}(x, \xi)\right| \leqq C_{\alpha \beta}\langle x\rangle^{-N} \quad(|\alpha|+|\beta| \leqq k),
$$

where $k$ can be chosen large enough, and $A$ is a F.I.Op.

$$
A f(x)=\int e^{i \phi(x . \xi)} c(x, \xi) f(\xi) d \xi .
$$

Here $c(x, \xi)$ satisfies

$$
\begin{array}{ll}
\left|D_{x}^{\alpha} D_{\xi}^{\beta} c(x, \xi)\right| \leqq C_{\alpha \beta m}\langle x\rangle^{-m}, & \text { (for any } m>0, \text { if } \hat{x} \cdot \hat{\xi}>-1+\varepsilon), \\
\left|D_{x}^{\alpha} D_{\xi}^{\beta} c(x, \xi)\right| \leqq C_{a \beta} & \text { (if } \hat{x} \cdot \hat{\xi}<-1+\varepsilon \text { ) }
\end{array}
$$

Choose a constant $\tilde{\mu}$ such that $-1+\varepsilon<\tilde{\mu}<\mu_{+}$and $C^{\infty}$-functions $\rho_{1}(t), \rho_{2}(t)$ such that $\rho_{1}(t)+\rho_{2}(t)=1$ for any $t, \quad \rho_{1}(t)=1$ if $t>\tilde{\mu}+3\left(\mu_{+}-\tilde{\mu}\right) / 4, \rho_{2}(t)=1$ for $t<\tilde{\mu}-$ $(\tilde{\mu}+1-\varepsilon) / 4$. Split $A$ into two parts: $A=A_{1}+A_{2}$, where

$$
A_{\jmath} f(x)=\int e^{i \dot{\varphi}(x, \xi)} c(x, \xi) \rho_{j}(\hat{x} \cdot \hat{\xi}) \hat{f}(\xi) d \xi, \quad j=1,2 .
$$

Since the symbols of $A_{1}$ and $P_{N}$ are rapidly decreasing in $x$, we can apply Lemma 2.1 (1) to see that

$$
\begin{array}{r}
\left\|\langle x\rangle^{s} A_{1} e^{-i t H_{0}} B_{+}(\lambda)^{*}\langle x\rangle^{s}\right\|+\left\|\langle x\rangle^{s} P_{N} e^{-\imath t H_{0}} B_{+}(\lambda) *\langle x\rangle^{s}\right\| \leqq C_{N}(1+t \sqrt{\lambda})^{-m(N)} . \\
t \geqq 0,
\end{array}
$$

where $m(N) \rightarrow \infty$ as $N \rightarrow \infty$. Applying Lemma 2.1 (2), we see that $\langle x\rangle^{s} A_{2} e^{-\imath t H_{0}}$ $B_{+}(\lambda)^{*}\langle x\rangle^{s}$ also has the desired decay rate. 
Passing to the Laplace transform in Lemma 2.5, we can show

Lemma 2.6. (1) For any $s \geqq 0$ and $\delta>1$, we have for any $\lambda>a_{0}>0$

$$
\left\|\langle x\rangle^{-(s+\delta)} A_{+} R_{0}(\lambda+i 0) B_{+}(\lambda) *\langle x\rangle^{s}\right\| \leqq C / \sqrt{\lambda} .
$$

(2) If $\mu_{+}>-1+\varepsilon$, we have for any $s \geqq 0$

$$
\left\|\langle x\rangle^{s}\left\langle D_{x}\right\rangle^{-1} G_{+} R_{0}(\lambda+i 0) B_{+}(\lambda) *\langle x\rangle^{s}\right\| \leqq C / \sqrt{\lambda},
$$

for any $\lambda>a_{0}>0$.

\section{§3. One Sided Localizations}

Let $A_{+}$and $G_{+}$be as in Definition 2.4. Since $\left(H-|\xi|^{2}\right) e^{2 \dot{c}(x, \xi)} a(x, \xi)=$ $e^{i \dot{\varphi}(x, \xi)} G(x, \xi)$, one can easily show that

$$
H A_{+}-A_{+} H_{0}=G_{+} \text {. }
$$

Multiplying both sides of (3.1) by $R(z)=(H-z)^{-1}$ and $R_{0}(z)=\left(H_{0}-z\right)^{-1}$, we have

$$
R(z) A_{+}=A_{+} R_{0}(z)-R(z) G_{+} R_{0}(z),
$$

which is the fundamental formula we use to derive the micro-local resolvent estimates.

We also prepare an estimate for the resolvent.

Lemma 3.1. Let $a_{0}>0$ be arbitrarily fixed. Then we have for any $s>1 / 2$

$$
\left\|\langle x\rangle^{-s}\left\langle D_{x}\right\rangle R(\lambda \pm i 0)\langle x\rangle^{-s}\right\|<C, \quad \lambda>a_{0},
$$

where $\left\langle D_{x}\right\rangle$ is a Ps. D. Op. with symbol $\langle\xi\rangle$ and $C$ is a constant independent of $\lambda>a_{0}$.

Indeed, this easily follows from (0.2) and the a-priori estimate given in [1], Theorem 1.4.

Lemma 3.2. Let $P_{ \pm}(\lambda)$ be the Ps. D. Op. with symbol $p_{ \pm}(x, \xi ; \lambda)$ satisfying (2.6)-(2.9). Then for any $s \geqq 0$ and $\delta>1$, we have

$$
\begin{aligned}
& \left\|\langle x\rangle^{-(s+\delta)} R(\lambda \pm i 0) P_{ \pm}(\lambda)\langle x\rangle^{s}\right\| \leqq C / \sqrt{\lambda}, \\
& \left\|\langle x\rangle^{-(s+\delta)} R(\lambda \pm i 0) P_{ \pm}(\lambda) *\langle x\rangle^{s}\right\| \leqq C / \sqrt{\lambda},
\end{aligned}
$$

where the constant $C$ is independent of $\lambda>a_{0}>0, a_{0}$ being an arbitrarily fixed constant.

Proof. First we show that (3.4) follows from (3.3). In fact, by [1], Theorem 2.4, for any $N \geqq 1$, there exist Ps. D. Op.'s $P_{ \pm}^{(N)}(\lambda)$ and $P_{N}(\lambda)$ such that $P_{ \pm}(\lambda) *=$ $P_{ \pm}^{(N)}(\lambda)+P_{N}(\lambda)$, the symbol of $P_{ \pm}^{(N)}(\lambda)$ satisfies $(2.6)-(2.9)$ and the symbol of $P_{N}(\lambda)$ 
satisfies

$$
\left|D_{x}^{\alpha} D_{\xi}^{\beta} p_{N}(x, \xi ; \lambda)\right| \leqq C_{\alpha \beta}\langle x\rangle^{-N-|\alpha|} .
$$

Since $R(\lambda \pm i 0) P_{ \pm}(\lambda) *=R(\lambda \pm i 0) P_{ \pm}^{(N)}(\lambda)+R(\lambda \pm i 0) P_{N}(\lambda)$, (3.4) immediately follows from (3.3) and (0.2).

Now, we prove (3.3) for $R(\lambda+i 0) P_{+}(\lambda)$. For $p_{+}(x, \xi ; \lambda)$, we choose $\varepsilon, d>0$ small enough so that $-1+\varepsilon<\mu_{+}$and $p_{+}(x, \xi ; \lambda)=0$ if $|\xi|>d$. For these $\varepsilon$ and $d$, we construct $\phi(x, \xi)$ and $a(x, \xi)$ as in Theorems 2.2 and 2.3. Then by Theorem 7.4 , for any $N \geqq 1$, there exist a F.I.Op. $B_{+}(\lambda)$ with symbol $b_{+}(x, \xi ; \lambda)$ satisfying (2.6)-(2.9) and a Ps.D. Op. $P_{N}(\lambda)$ with symbol satisfying (3.5) such that

$$
P_{+}(\lambda)=A_{+} B_{+}(\lambda) *+P_{N}(\lambda) .
$$

Using (3.2) and (3.6), we have

$$
\begin{aligned}
R(\lambda+i 0) P_{+}(\lambda)= & R(\lambda+i 0) P_{N}(\lambda)+A_{+} R_{0}(\lambda+i 0) B_{+}(\lambda) * \\
& -R(\lambda+i 0) G_{+} R_{0}(\lambda+i 0) B_{+}(\lambda) * .
\end{aligned}
$$

The first term of the right-hand side of (3.7) is easily seen to satisfy (3.3) by virtue of (0.2). To the second term, we apply Lemma 2.6, (1). To the third term, we have only to apply Lemma 2.6, (2) and Lemma 3.1.

In order to treat the low frequency term, we introduce the following class of Ps. D. Op.'s.

Definition 3.3. Let $\lambda>a_{0}>0$. A Ps. D. Op. $Q(\lambda)$ belongs to $\mathcal{S}_{0}(\lambda)$ if its symbol $q(x, \xi ; \lambda)$ satisfies

$$
\left|D_{x}^{\alpha} D_{\xi}^{\beta} q(x, \xi ; \lambda)\right| \leqq C_{\alpha \beta}\langle x\rangle^{-|\alpha|},
$$

where the constant $C_{\alpha \beta}$ is independent of $\lambda>a_{0}>0$,

$$
q(x, \xi ; \lambda)=0 \quad \text { if } \quad|\xi|>\frac{3}{4} \sqrt{\lambda} .
$$

Lemma 3.4. Let $Q(\lambda) \in \mathcal{S}_{0}(\lambda)$. Then for any $s>1 / 2$

$$
\left\|\langle x\rangle^{s} Q(\lambda) R(\lambda \pm i 0)\langle x\rangle^{-s}\right\| \leqq C / \lambda
$$

where the constant $C$ is independent of $\lambda>a_{0}>0$.

Proof. Let $P(\lambda)=Q(\lambda) R_{0}(\lambda \pm i 0)$, which is the Ps.D.Op. with symbol $q(x, \xi ; \lambda)\left(|\xi|^{2}-\lambda\right)^{-1}$. Then we have by the resolvent equation

$$
Q(\lambda) R(\lambda \pm i 0)=P(\lambda)-P(\lambda) V R(\lambda \pm i 0) .
$$

Since $Q(\lambda) \in \mathcal{S}_{0}(\lambda)$, one can easily show that the symbol $p(x, \xi ; \lambda)$ of $P(\lambda)$ satisfies

$$
\left|D_{x}^{\alpha} D_{\xi}^{\beta} p(x, \xi ; \lambda)\right| \leqq C_{\alpha, 3} \lambda^{-1}\langle x\rangle^{-|\alpha|},
$$


$C_{\alpha \beta}$ being a constant independent of $\lambda>a_{0}>0$. By the asymptotic expansion ([1], Theorem 2.3), for any $N \geqq 1$, there exist Ps. D. Op.'s $P_{N}(\lambda) \in \mathcal{S}_{0}(\lambda)$ and $Q_{N}(\lambda)$ such that $P(\lambda) V=\langle\lambda\rangle^{-\varepsilon_{0}} P_{N}(\lambda)+Q_{N}(\lambda)$, where the symbol of $P_{N}(\lambda)$ has the same estimates as in $(3.9)$ and the symbol of $q_{N}(x, \xi ; \lambda)$ of $Q_{N}(\lambda)$ satisfies

$$
\left|D_{x}^{a} D_{\xi}^{\beta} q_{N}(x, \xi ; \lambda)\right| \leqq C_{a \beta} \lambda^{-1}\langle x\rangle^{-N-|a|}, \quad \lambda>a_{0}>0 .
$$

In view of $(3.8)$, we have

$$
Q(\lambda) R(\lambda \pm i 0)=P(\lambda)-\langle x\rangle^{-\varepsilon_{0}} P_{N}(\lambda) R(\lambda \pm i 0)-Q_{N}(\lambda) R(\lambda \pm i 0) .
$$

Therefore, if $r>1 / 2, r \geqq s$, choosing $N$ large enough, we have noting (0.2) and (3.9)

$$
\left\|\langle x\rangle^{s} Q(\lambda) R(\lambda \pm i 0)\langle x\rangle^{-r}\right\| \leqq C\left(1 / \lambda+\left\|\langle x\rangle^{s-\varepsilon_{0}} P_{N}(\lambda) R(\lambda \pm i 0)\langle x\rangle^{-r}\right\|\right) .
$$

Since $P_{N}(\lambda) \in \mathcal{S}_{0}(\lambda)$, one can use the above inequality to estimate the term $P_{N}(\lambda) R(\lambda \pm i 0)$ to obtain

$$
\left\|\langle x\rangle^{s} Q(\lambda) R(\lambda \pm i 0)\langle x\rangle^{-r}\right\| \leqq C\left(1 / \lambda+\left\|\langle x\rangle^{s-2 \varepsilon_{0}} \widetilde{P}_{N}(\lambda) R(\lambda \pm i 0)\langle x\rangle^{-r}\right\|\right)
$$

for some $P_{N}(\lambda) \in \mathcal{S}_{0}(\lambda)$. Repeating this procedure, we obtain the lemma.

Theorem 1 in the introduction now easily follows from Lemmas 3.2 and 3.4, if we split the symbol of $P_{ \pm}$suitably. More precisely, we split $p_{ \pm}(x, \xi)=$ $p_{ \pm}(x, \xi) \phi_{0}(\xi / \sqrt{\lambda})+p_{ \pm}(x, \xi) \phi^{\infty}(\xi / \sqrt{\lambda})$, where $\phi_{0}(\xi), \phi^{\infty}(\xi) \in C^{\infty}\left(\mathbb{R}^{n}\right), \phi_{0}(\xi)+\phi^{\infty}(\xi)=1$, $\phi_{0}(\xi)=1$ if $|\xi|<1 / 2, \phi^{\infty}(\xi)=1$ if $|\xi|>3 / 4$.

\section{§4. Two Sided Localizations}

We shall prove Theorem 2. As the first step, we show the following

Lemma 4.1. Let $P_{-}$be the Ps. D. Op. with symbol satisfying (0.3)-(0.5). Let $P_{+}(\lambda)$ be the Ps. D. Op. with symbol $p_{+}(x, \xi ; \lambda)$ satisfying (2.6)-(2.9). Assume that $\mu_{+}>\mu_{-}$. Then we have for any $s>0$

$$
\left\|\langle x\rangle^{s} P_{-} R(\lambda+i 0) P_{+}(\lambda)\langle x\rangle^{s}\right\| \leqq C / \sqrt{\lambda}, \quad \lambda>a_{0}>0 .
$$

Proof. As has been discussed in the proof of Lemma 3.2, for any $N \geqq 1$, there exist a F. I. Op. $B_{+}(\lambda)$ with symbol $b_{+}(x, \xi ; \lambda)$ satisfying $(2.6)-(2.9)$ and a Ps. D. Op. $P_{N}(\lambda)$ with symbol satisfying (3.5) such that $P_{+}(\lambda)=A_{+} B_{+}(\lambda) *+P_{N}(\lambda)$. In view of (3.7), we have

$$
\begin{aligned}
P_{-} R(\lambda+i 0) P_{+}(\lambda)= & P_{-} R(\lambda+i 0) P_{N}(\lambda)+P_{-} A_{+} R_{0}(\lambda+i 0) B_{+}(\lambda) * \\
& -P_{-} R(\lambda+i 0) G_{+} R_{0}(\lambda+i 0) B_{+}(\lambda)^{*}
\end{aligned}
$$

Taking $N$ large enough, we see that the first term of the right-hand side of (4.1) has the desired property by virtue of Theorem 1. Using Lemma 2.6 (2), we see that the third term has the desired property î́ we note that 


$$
\left\|\langle x\rangle^{s} P_{-} R(\lambda+i 0)\left\langle D_{x}\right\rangle\langle x\rangle^{-(s+\delta)}\right\| \leqq C, \quad \lambda>a_{0}>0,
$$

for $s \geqq 0, \delta>1$, which follows from Theorem 1 and [1], Theorem 1.4.

We consider the second term. By Theorem 6.2 in Section 6 , for large $N>0$, $P_{-} A_{+}$is split into two parts: $P_{-} A_{+}=A_{1}+Q_{N}$, where $Q_{N}$ is a Ps. D.Op. with symbol $q_{N}(x, \xi)$ such that

$$
\left|D_{x}^{\alpha} D_{\xi}^{\beta} q_{N}(x, \xi)\right| \leqq C_{\alpha \beta}\langle x\rangle^{-N} \quad(|\alpha|+|\beta| \leqq k),
$$

where $k$ can be chosen large enough, and $A_{1}$ is an F.I.Op.

$$
\begin{aligned}
& A_{1} f(x)=\int e^{i \dot{\phi}(x, \xi)} c(x, \xi) \hat{f}(\xi) d \xi, \\
& \left|D_{x}^{\alpha} D_{\xi}^{\beta} c(x, \xi)\right| \leqq C_{\alpha,}\langle x\rangle^{-|\alpha|}, \\
& c(x, \xi)=0 \quad \text { if } \hat{x} \cdot \hat{\xi}>\mu_{-} .
\end{aligned}
$$

Therefore, $A_{1} e^{-i t H_{0}} B_{+}(\lambda) *$ has the same decay property as in Lemma 2.1 (2). Passing to the Laplace transform, we can show that $A_{1} R_{0}(\lambda+i 0) B_{+}(\lambda) *$ has the desired property. Using Lemma 2.1 (1), one can treat $Q_{N} R_{0}(\lambda+i 0) B_{+}(\lambda) *$ similarly.

Lemma 4.2. Let $P_{-}$be the Ps. D. Op. with symbol satisfying (0.3)-(0.5). Let $Q_{+}(\lambda)$ be the Ps.D.Op. with symbol $q_{+}(x, \xi ; \lambda)$ satisfying (2.6), (2.8). (2.9) and

$$
q_{+}(x, \xi ; \lambda)=0 \quad \text { if } \quad|\xi|>\frac{3}{4} \sqrt{\lambda}, \quad \lambda>a_{0}>0 .
$$

Assume that $\mu_{+}>\mu_{-}$. Then we have for any $s>0$

$$
\begin{array}{ll}
\left\|\langle x\rangle^{s} P_{-} R(\lambda+i 0) Q_{+}(\lambda) *\langle x\rangle^{s}\right\| \leqq C / \sqrt{\lambda}, & \lambda>a_{0}>0, \\
\left\|\langle x\rangle^{s} P_{-} R(\lambda+i 0) Q_{+}(\lambda)\langle x\rangle^{s}\right\| \leqq C / \sqrt{\lambda}, & \lambda>a_{0}>0 .
\end{array}
$$

Proof. First we derive (4.4) from (4.3). By [1], Theorem 2.4, for any $N \geqq 1$, there exist Ps.D.Op.'s $Q_{N}(\lambda)$ and $P_{N}(\lambda)$ such that $Q_{+}(\lambda) *=Q_{N}(\lambda)+P_{N}(\lambda)$, the symbol of $Q_{N}(\lambda)$ satisfies (2.6), (2.8), (2.9), (4.2) and the symbol of $P_{N}(\lambda)$ satisfies (3.5). Since $P_{-} R(\lambda+i 0) Q_{+}(\lambda)=P_{-} R(\lambda+i 0) Q_{N}(\lambda) *+P_{-} R(\lambda+i 0) P_{N}(\lambda) *$, (4.4) follows from (4.3) and Theorem 1, if we choose $N$ large enough.

We turn to the proof of (4.3). Let $P_{+}(\lambda)$ be the Ps.D.Op. with symbol $q_{+}(x, \xi ; \lambda)\left(|\xi|^{2}-\lambda\right)^{-1}$. Then, as is easily seen, $R_{0}(\lambda+i 0) Q_{+}(\lambda) *=P_{+}(\lambda) *$. The resolvent equation implies that

$$
P_{-} R(\lambda+i 0) Q_{+}(\lambda) *=P_{-} P_{+}(\lambda) *-P_{-} R(\lambda+i 0) V P_{+}(\lambda) * .
$$

Again using [1], Theorem 2.3, for any $N \geqq 1$, there exist Ps. D. Op.'s $P^{(+)}(\lambda)$ and $P_{N}(\lambda)$ such that $P_{+} V=\langle x\rangle^{-\varepsilon_{0}} P^{(+)}(\lambda)+P_{N}(\lambda)$, where the symbol of $P^{(+)}(\lambda)$ satisfies (2.6), (2.8), (2.9), (4.2) and the symbol of $P_{N}(\lambda)$ satisfies (3.5). Then, (4.5) can be written as 


$$
P_{-} R(\lambda+i 0) Q_{+}(\lambda) *=P_{-} P_{+}(\lambda) *-P_{-} R(\lambda+i 0) P_{N}(\lambda)-P_{-} R(\lambda+i 0) P^{(+)}(\lambda)\langle x\rangle^{-\varepsilon_{0}} .
$$

Using [1], Theorems 2.3 and 2.4 and the condition $\mu_{+}>\mu_{-}$, one can show that for any $s>0$

$$
\left\|\langle x\rangle^{s} P_{-} P_{+}(\lambda) *\langle x\rangle^{s}\right\| \leqq C / \lambda, \quad \lambda>a_{0}>0 .
$$

In view of Theorem 1 , we have for any $s>0$, taking $N$ large enough,

$$
\left\|\langle x\rangle^{s} P_{-} R(\lambda+i 0) P_{N}(\lambda) *\langle x\rangle^{s}\right\| \leqq C / \sqrt{\lambda}, \quad \lambda>a_{0}>0 .
$$

Therefore, from (4.6) (4.8), we have for any $s>0$ and $r \in \mathbb{R}^{1}$,

$$
\left\|\langle x\rangle^{s} P_{-} R(\lambda+i 0) Q_{+}(\lambda) *\langle x\rangle^{r}\right\| \leqq C\left(\lambda^{-1 / 2}+\left\|\langle x\rangle^{s} P_{-} R(\lambda+i 0) P^{(+)}(\lambda) *\langle x\rangle^{r-\varepsilon_{0}}\right\|\right) .
$$

Since $P^{(+)}(\lambda)$ satisfies (2.6), (2.8), (2.9) and (4.2), one can use (4.9) itself to estimate the right-hand side of (4.9). Thus we can replace $r-\varepsilon_{0}$ in (4.9) by $r-2 \varepsilon_{0}$. Repeating this procedure, we finally obtain

$$
\left\|\langle x\rangle^{s} P_{-} R(\lambda+i 0) Q_{+}(\lambda) *\langle x\rangle^{r}\right\| \leqq C\left(\lambda^{-1 / 2}+\left\|\langle x\rangle^{s} P_{-} R(\lambda+i 0)\langle x\rangle^{-s-2}\right\|\right) \leqq C / \sqrt{\lambda},
$$

where we have used Theorem 1.

Remark 4.3. Let $P_{ \pm}$be Ps. D. Op.'s with symbols satisfying $(0.3) \sim(0.5)$. Let $B$ be the Ps. D. Op. with symbol $\psi(x, \xi)$ such that $\mid D_{x}^{\alpha} D_{\xi}^{\beta} \psi(x, \xi) ! \leqq C_{\alpha \beta}\langle x\rangle^{-|\alpha|}$ and $\psi(x, \xi)=0$ if $|\xi|>2 \varepsilon / 3$, where $\varepsilon$ is the constant specified in (0.4). Assume that $a_{0}>\varepsilon^{2}$. Then by the same arguments as above, one can show that for any $s>0$

$$
\begin{array}{ll}
\left\|\langle x\rangle^{s} P_{\mp} R(\lambda \pm i 0) B^{*}\langle x\rangle^{s}\right\| \leqq C / \sqrt{\lambda}, & \lambda>a_{0}>0, \\
\left\|\langle x\rangle^{s} P_{\mp}^{*} R(\lambda \pm i 0) B^{*}\langle x\rangle^{s}\right\| \leqq C / \sqrt{\lambda}, & \lambda>a_{0}>0 .
\end{array}
$$

These estimates will be used in $\S 5$.

Now, in order to prove Theorem 2 in the introduction, we have only to split the symbol of $P_{ \pm}$suitably and apply Lemmas 4.1 and 4.2.

\section{$\S 5$. Micro-local Approximation of Resolvents}

In this section, we shall prove Theorem 3. Choose $C^{\infty}$-functions $\phi(\xi), \phi(\xi)$ such that $\phi(\xi)+\phi(\xi)=1, \phi(\xi)=1$ for $|\xi|>2 \varepsilon / 3, \phi(\xi)=1$ for $|\xi|<\varepsilon / 3$. Let $A, B$ be Ps. D. Op.'s with symbols $\phi(\xi), \phi(\xi)$, respectively. Then, letting $\tilde{V}_{\jmath}=V-V_{\jmath}$,

$$
\begin{aligned}
& P_{-}\left(R_{\jmath}(\lambda+i 0)-R(\lambda+i 0)\right) P_{+} \\
= & P_{-} R(\lambda+i 0) \tilde{V}_{\jmath} A R_{\jmath}(\lambda+i 0) P_{+}+P_{-} R(\lambda+i 0) \tilde{V}_{\jmath} B R_{\jmath}(\lambda+i 0) P_{+} .
\end{aligned}
$$

The second term of the right-hand side is estimated as follows:

$$
\begin{aligned}
& \left\|\langle x\rangle^{s} P_{-} R(\lambda+i 0) \tilde{V}, B R_{\jmath}(\lambda+i 0) P_{+}\langle x\rangle^{s}\right\| \\
\leqq & \left\|\langle x\rangle^{s} P_{-} R(\lambda+i 0)\langle x\rangle^{-s-2}\right\| \cdot\left\|\tilde{V}_{\jmath}\right\| \cdot\left\|\langle x\rangle^{s+2} B R_{\jmath}(\lambda+i 0) P_{+}\langle x\rangle^{s}\right\| .
\end{aligned}
$$


The first factor is bounded by $C / \sqrt{\lambda}$ by Theorem 1 . The second factor is bounded by $C j^{-s_{0}}$. The third factor is bounded by $C / \sqrt{\lambda}$ by virtue of Remark 4.3, where the constant $C$ is independent of $j$. Therefore, we have

$$
\left\|\langle x\rangle^{s} P_{-} R(\lambda+i 0) \tilde{V}_{\jmath} B R_{\jmath}(\lambda+i 0) P_{+}\langle x\rangle^{s}\right\| \leqq C j^{-\varepsilon_{0}} \lambda^{-1} .
$$

Next we consider the first term of the right-hand side of (5.1). Choose $C^{\infty}$ functions $\rho_{ \pm}(t)$ such that $\rho_{+}(t)+\rho_{-}(t)=1, \rho_{+}(t)=1$ for $t>\mu_{-}+2\left(\mu_{+}-\mu_{-}\right) / 3, \rho_{-}(t)=1$ for $t<\mu_{-}+\left(\mu_{+}-\mu_{-}\right) / 3$. Let $A_{\jmath_{ \pm}}$be Ps. D. Op.'s with symbols $\tilde{V}_{\jmath}(x) \rho_{ \pm}(\hat{x} \cdot \hat{\xi}) \phi(\xi)$. Then

$$
\begin{aligned}
& P_{-} R(\lambda+i 0) \tilde{V}_{\jmath} A R_{\jmath}(\lambda+i 0) P_{+} \\
= & P_{-} R(\lambda+i 0) A_{j+} R_{\jmath}(\lambda+i 0) P_{+}+P_{-} R(\lambda+i 0) A_{\jmath} R_{\jmath}(\lambda+i 0) P_{+} .
\end{aligned}
$$

The first term of the right-hand side of (5.3) is estimated as follows :

$$
\begin{aligned}
& \left\|\langle x\rangle^{s} P_{-} R(\lambda+i 0) A_{\jmath+} R_{\jmath}(\lambda+i 0) P_{+}\langle x\rangle^{s}\right\| \\
\leqq & \left\|\langle x\rangle^{s} P_{-} R(\lambda+i 0) A_{\jmath+}\langle x\rangle^{s+2}\right\| \cdot\left\|\langle x\rangle^{-s-2} R_{\jmath}(\lambda+i 0) P_{+}\langle x\rangle^{s}\right\| .
\end{aligned}
$$

The second factor is bounded by $C / \sqrt{\lambda}$ by Theorem 1 , the constant $C$ being independent of $j$. Theorem 2 implies that the first factor is bounded by $C j^{-s_{0}} / \sqrt{\lambda}$, where we have used an estimate for the commutator of $\tilde{V}_{j}$ and the Ps.D.Op. with symbol $\phi(x) \rho_{+}(\hat{x} \cdot \hat{\xi}) \phi(\xi)$.

The second term of the right-hand side of (5.3) is estimates as follows:

$$
\begin{aligned}
& \left\|\langle x\rangle^{s} P_{-} R(\lambda+i 0) A_{j-} R_{\jmath}(\lambda+i 0) P_{+}\langle x\rangle^{s}\right\| \\
\leqq & \left\|\langle x\rangle^{s} P_{-} R(\lambda+i 0)\langle x\rangle^{-s-2}\right\| \cdot\left\|\langle x\rangle^{s+2} A_{\jmath} R_{\jmath}(\lambda+i 0) P_{+}\langle x\rangle^{s}\right\| \\
\leqq & C j^{-\varepsilon_{0}} \lambda^{-1},
\end{aligned}
$$

where we have used Theorems 1 and 2. Thus we have

$$
\left\|\langle x\rangle^{s} P_{-} R(\lambda+i 0) \tilde{V}_{j} A R_{\jmath}(\lambda+i 0) P_{+}\langle x\rangle^{s}\right\| \leqq C j^{-\varepsilon_{0}} \lambda^{-1} .
$$

Theorem 3 directly follows from (5.1), (5.2) and (5.4).

\section{$\S$ 6. Products of Fourier Integral Operators and Pseudo-differential Operators}

In this section, we derive a formula for the product of F.I.Op.'s and Ps. D. Op.'s. Our treatment is slightly different from the standard one (see e.g. $[5])$.

First we introduce a class of symbols.

Definition 6.1. Let $\sigma, m \in \mathbb{R}^{1} . \quad S(\sigma, m)$ is the set of $C^{\infty}\left(\boldsymbol{R}^{n} \times \mathbb{R}^{n}\right)$-functions $p(x, \xi)$ such that 


$$
\left|D_{x}^{\alpha} D_{\xi}^{\beta} p(x, \xi)\right| \leqq C_{\alpha \beta}\langle x\rangle^{\sigma-|\alpha|\langle\xi\rangle^{m}}
$$

Let $\dot{\phi}(x, \xi)$ be a real $C^{\infty}\left(\boldsymbol{R}^{n} \times \boldsymbol{R}^{n}\right)$-function such that

$$
\left\{\begin{array}{l}
\phi(x, \xi)=x \xi+Y(x, \xi), \\
\left|D_{x}^{\alpha} D_{\xi}^{\beta} Y(x, \xi)\right| \leqq C_{\alpha \beta}\langle x\rangle^{1-|\alpha|-\varepsilon_{0}}, \quad\left(0<\varepsilon_{0}<1\right) .
\end{array}\right.
$$

For $a(x, \xi) \in S\left(\sigma_{1}, m_{1}\right)$ and $p(x, \xi) \in S\left(\sigma_{2}, m_{2}\right)$, we define a F.I.Op. $A$ and a Ps. D. Op. $P$ by

$$
\begin{aligned}
& A f(x)=\int e^{i \phi(x, \xi)} a(x, \xi) \hat{f}(\xi) d \xi, \\
& P f(x)=\int e^{i x \xi} p(x, \xi) \hat{f}(\xi) d \xi .
\end{aligned}
$$

Note that $A$ is $L^{2}$-bounded if $\sigma_{1} \leqq 0$ and $m_{1} \leqq 0$ ([2], Lemma 3.3).

Theorem 6.2. Let $a(x, \xi) \in S\left(\sigma_{1}, m_{1}\right)$ and $p(x, \xi) \in S\left(\sigma_{2}, m_{2}\right)$. Then for any integers $N, k \geqq 1$, there exist $a$ F.I.Op. $A_{1}$ and $a$ Ps. D.Op. $P_{N}$ such that

$$
P A=A_{1}+P_{N},
$$

where

$$
\begin{aligned}
& A_{1} f(x)=\int e^{\imath \dot{\psi}(x, \xi)} a_{1}(x, \xi) \hat{f}(\xi) d \xi, \\
& P_{N} f(x)=\int e^{i x \xi} p_{N}(x, \xi) \hat{f}(\xi) d \xi,
\end{aligned}
$$

and $a_{1}(x, \xi)$ consists of a finite sum of the following terms:

$$
\begin{array}{r}
\left(\partial_{\xi}^{\alpha} p(x, \xi)\right) \cdot\left(D_{x}^{\beta_{1}} Y(x, \xi) \cdots D_{x}^{\beta} t Y(x, \xi)\right) D_{x}^{\beta} a(x, \xi), \\
\left|\beta_{1}\right|+\cdots+\left|\beta_{t}\right|+|\beta|=|\alpha|,
\end{array}
$$

$p_{N}(x, \xi)$ satisfies

$$
\sum_{|\beta| \leqq k}\left|D_{x}^{\alpha} D_{\xi}^{\beta} p_{N}(x, \xi)\right| \leqq C_{a k N}\langle x\rangle^{-N}\langle\xi\rangle^{m_{1}+m_{2}} 。
$$

Proof. Let $b(x, \xi)=e^{i Y(x, \xi)} a(x, \xi)$. Then $A$ can be regarded as a Ps. D. Op. with symbol $b(x, \xi)$. Applying the well-known formula of the asymptotic expansion of the symbol of the product of Ps. D. Op.'s (see Kumanogo [4], p. 75), we see that the symbol of $P A$ (regarded as a Ps. D. Op.) is given by

$$
\begin{aligned}
& q_{M}(x, \xi)+r_{M}(x, \xi), \\
& q_{M}(x, \xi)=\sum_{|\alpha|<M} \frac{1}{\alpha !} \partial_{\xi}^{a} p(x, \xi) \cdot D_{x}^{a} b^{\prime}(x, \xi), \\
& r_{M}(x, \xi)=M \sum_{|\gamma|=M} \frac{1}{\gamma !} O s-\iiint_{0}^{1} d \theta(1-\theta)^{M-1} e^{-\imath y r} \partial_{\xi}^{r} p(x, \xi+\theta \eta) D_{x}^{r} b\left(x+y^{\prime}, \xi\right) d \eta d \eta .
\end{aligned}
$$


Define $a_{1}(x, \xi)=e^{-i Y(x, \xi)} q_{M}(x, \xi)$. Then the Ps. D. Op. with symbol $q_{M}(x, \xi)$ can be written as (6.6). One can also see that $a_{1}(x, \xi)$ consists of the sum of the terms like (6.8). $r_{M}(x, \xi)$ is estimated as follows. Choose $M$ large enough. Then if $|\gamma|=M$, we have by our assumptions

$$
\begin{aligned}
& \sum_{\mid \beta_{1} \leqq k}\left|D_{x}^{\alpha} D_{\xi}^{\beta} p(x, \xi)\right| \leqq C_{\alpha k N}\langle x\rangle^{\sigma_{2}-|\alpha|}\langle\xi\rangle^{m_{2}}, \\
& \sum_{|,| \leqq k}\left|D_{x}^{\alpha} D_{\xi}^{\beta} b(x, \xi)\right| \leqq C_{\alpha k N}\langle x\rangle^{\sigma_{1}-N}\langle\xi\rangle^{m_{1}} .
\end{aligned}
$$

With this in mind, one can argue as in [4], pp. $69 \sim 72$ to see that $r_{M}(x, \xi)$ satisfies (6.9).

\section{$\S 7$. Reduction of Fourier Integral Operators to Pseudo-differential Operators}

In this section, we shall derive a formula for transforming the product of F.I.Op.'s into a Ps. D. Op. We begin with the following lemma.

Lemma 7.1 (Left simplified symbol). Let $P$ be $a$ Ps. D. Op. such that

$$
\begin{aligned}
& P f(x)=\iint e^{i(x-y) \xi} a(x, \xi, y) f(y) d y d \xi, \\
& \left|D_{x}^{\alpha} D_{\xi}^{\beta} D_{y}^{\gamma} a(x, \xi, y)\right| \leqq C_{\alpha \beta \gamma}\langle x\rangle^{-|\alpha|}\langle y\rangle^{-|\gamma|} .
\end{aligned}
$$

Define $p(x, \xi)$ by

$$
p(x, \xi)=O s-\iint e^{-i y \eta} a(x, \xi+\eta, x+y) d y d \eta .
$$

Then $P$ is a Ps. D. Op. with symbol $p(x, \xi)$. Furthermore, for any $N \geqq 1$

$$
\begin{aligned}
& p(x, \xi)=\left.\sum_{|\alpha|<N} \frac{1}{\alpha !} \partial_{\xi}^{\alpha} D_{y}^{\alpha} a(x, \xi, y)\right|_{y=x}+r_{N}(x, \xi), \\
& \left|D_{x}^{\alpha} D_{\xi}^{\beta} r_{N}(x, \xi)\right| \leqq C_{a \beta N}\langle x\rangle^{-N-|\alpha|} .
\end{aligned}
$$

The proof is routine, hence is omitted (see Kumanogo [4], pp. 73, 76).

Let us rewrite $A_{+} B_{+}(\lambda) *$ into a Ps. D. Op., where $A_{+}$and $B_{+}(\lambda) *$ have been introduced in Definition 2.4. By definition

$$
\begin{aligned}
& A_{+} B_{+}(\lambda) * f(x)=\iint e^{i S(x, \xi, y)} a(x, \xi) \overline{b_{+}(y, \xi ; \lambda)} f(y) d y d \xi, \\
& S(x, \xi, y)=\phi(x, \xi)-\phi(y, \xi) .
\end{aligned}
$$

Let $\chi_{0}(x) \in C_{0}^{\infty}\left(\boldsymbol{R}^{n}\right)$ be such that $\chi_{0}(x)=1$ for $|x|<1, \chi_{0}(x)=0$ for $|x|>2$. We set $\chi_{\infty}(x)=1-\chi_{0}(x)$. Choose $\chi_{ \pm}(t) \in C^{\infty}\left(\boldsymbol{R}^{1}\right)$ such that $\chi_{+}(t)+\chi_{-}(t)=1, \chi_{+}(t)=0$ if $t<-1 / 2, \quad \chi_{-}(t)=0$ if $t>1 / 2$. We split $A_{+} B_{+}(\lambda) *$ into three parts: $A_{+} B_{+}(\lambda) *=$ $Q_{1}(\lambda)+Q_{2}(\lambda)+Q_{3}(\lambda)$, where 


$$
\begin{aligned}
& Q_{1}(\lambda) f(x)=\iint e^{i S(x, \xi, y)} a(x, \xi) \overline{b_{+}(y, \xi ; \lambda)} \chi_{+}(\hat{x} \cdot \hat{y}) \chi_{\infty}(y) f(y) d y d \xi, \\
& Q_{2}(\lambda) f(x)=\iint e^{i S(x, \xi, y)} a(x, \xi) \overline{b_{+}(y, \xi ; \lambda)} \chi_{-}(\hat{x} \cdot \hat{y}) \chi_{\infty}(y) f(y) d y d \xi . \\
& Q_{3}(\lambda) f(x)=\iint e^{i S(x, \xi, y)} a(x, \xi) \overline{b_{+}(y, \xi ; \lambda)} \chi_{0}(y) f(y) d y d \xi .
\end{aligned}
$$

By the mean value theorem,

$$
S(x, \xi, y)=\langle x-y, Z(x, \xi, y)\rangle, \quad Z(x, \xi, y)=\int_{0}^{1}\left(\nabla_{x} \phi\right)(t x+(1-t) y, \xi) d t .
$$

Let us consider the map: $\xi \rightarrow Z(x, \xi, y)$. In view of Theorem 2.2 (3), we have

$$
\left|\frac{\partial}{\partial \xi} Z(x, \xi, y)-I\right|<1 / 2 \text {. }
$$

On the support of $a(x, \xi) \overline{b_{+}(y, \xi ; \lambda)} \chi_{+}(\hat{x} \cdot \hat{y}) \chi_{\infty}(y)$,

$$
\left|D_{x}^{\alpha} D_{\xi}^{\beta} D_{y}^{\gamma}(Z(x, \xi, y)-\xi)\right| \leqq C_{\alpha \beta \gamma}\langle\xi\rangle^{-1}\langle x\rangle^{-\varepsilon_{0} / 2-|\alpha|}\langle y\rangle^{-\varepsilon_{0} / 2-|\gamma|} .
$$

In fact, in this case $\hat{x} \cdot \hat{y}>-1 / 2$. Therefore $|t x+(1-t) y| \geqq C(t|x|+(1-t)|y|)$ for $0 \leqq t \leqq 1$, with a constant $C>0$. Thus we have using Theorem 2.2 (2),

$$
\begin{aligned}
& \mid D_{x}^{\alpha} D_{\xi}^{\beta} D_{y}^{\gamma}(Z(x, \xi, y)-\xi) ! \\
\leqq & C_{\alpha \beta_{i}}\langle\xi\rangle^{-1} \int_{0}^{1} t^{|a|}(1-t)^{|\gamma|}(1+t|x|+(1-t)|y|)^{-\varepsilon_{0}-|\alpha|-|\gamma|} d t \\
\leqq & C_{\alpha \beta \gamma}\langle\xi\rangle^{-1}\left[\int_{0}^{1} t^{2|\alpha|}(1+t|x|)^{-\varepsilon_{0}-2|\alpha|} d t\right]^{1 / 2} \times\left[\int_{0}^{1} s^{2|\gamma|}(1+s|y|)^{-s_{0}-2|\gamma|} d s\right]^{1 / 2} \\
\leqq & C_{\alpha \beta \gamma}\langle\xi\rangle^{-1}\langle x\rangle^{-\varepsilon_{0} / 2-|\alpha|\langle y\rangle^{-\varepsilon_{0} / 2-|\gamma|}},
\end{aligned}
$$

which proves (7.4). (7.3) and (7.4) imply that the map $\xi \rightarrow Z(x, \xi, y)$ defines a differomorphism on $\boldsymbol{R}^{n}$ and that its inverse $\Psi$ satisfies

$$
\left|D_{x}^{\alpha} D_{\xi}^{\beta} D_{y}^{\gamma}(\Psi(x, \xi, y)-\xi)\right| \leqq C_{a \beta \gamma}\langle\xi\rangle^{-1}\langle x\rangle^{-s_{0} / 2-\mid \alpha_{1}}\langle y\rangle^{-\varepsilon_{0} / 2-|\gamma|},
$$

on the support of $a(x, \Psi(x, \xi, y)) \overline{b_{+}(y, \Psi(x, \xi, y) ; \lambda)} \chi_{+}(\hat{x} \cdot \hat{y}) \chi_{\infty}(y)$. Letting $J(x, \xi, y)$ $=\operatorname{det}\left(\partial_{\xi} \Psi(x, \xi, y)\right)$, we have

$$
\begin{aligned}
& Q_{1}(\lambda) f(x)=\iint e^{i(x-y) \xi} c(x, \xi, y ; \lambda) f(y) d y d \xi, \\
& c(x, \xi, y ; \lambda)=a(x, \Psi(x, \xi, y)) \overline{b_{+}(y, \Psi(x, \xi, y) ; \lambda)} \chi_{+}(\hat{x} \cdot \hat{y}) \chi_{\infty}(y) J(x, \xi, y) .
\end{aligned}
$$

We now make use of Lemma 7.1 to see that $Q_{1}(\lambda)$ is a Ps.D.Op. with symbol $q(x, \xi ; \lambda)$ having the following asymptotic expansion

$$
\begin{aligned}
& q(x, \xi ; \lambda)=\left.\sum_{|\alpha|<N} \frac{1}{\alpha !} \partial_{\xi}^{\alpha} D_{y}^{\alpha} c(x, \xi, y ; \lambda)\right|_{y=x}+q_{N}(x, \xi ; \lambda), \\
& \left|D_{x}^{\alpha} D_{\xi}^{\beta} q_{N}(x, \xi ; \lambda)\right| \leqq C_{\alpha \beta}\langle x\rangle^{-N-|a|} .
\end{aligned}
$$


In particular, the first term of the asymptotic expansion is $c(x, \xi, x ; \lambda)$. In view of (7.5), we have by the Taylor expansion

$$
c(x, \xi, x ; \lambda)=\sum_{m=0}^{N} c_{m}(x, \xi ; \lambda),
$$

where $c_{0}(x, \xi ; \lambda)=a(x, \xi) \overline{b_{+}(x, \xi ; \lambda)}$ and each $c_{m}(x, \xi ; \lambda)$ consists of derivatives

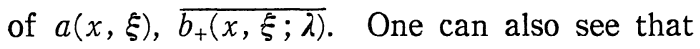

$$
\left|D_{x}^{\alpha} D_{\xi}^{\beta} c_{m}(x, \xi ; \lambda)\right| \leqq C_{\alpha \beta}\langle x\rangle^{-m \varepsilon_{0}-|\alpha|} .
$$

Let $d, \varepsilon>0$ be the constants specified in Theorem 2.3 (1). Assume that

$$
b_{+}(x, \xi ; \lambda)=0 \text { if }|\xi|<d \text { or } \hat{x} \cdot \hat{\xi}<\mu_{+}, \mu_{+}>-1+\varepsilon .
$$

Then, since $a(x, \xi)=1+O\left(|x|^{-\varepsilon_{0}}\right)$ on the support of $b_{+}(x, \xi ; \lambda)$, we have

$$
c_{0}(x, \xi ; \lambda)=\overline{b_{+}(x, \xi ; \lambda)}+\langle x\rangle^{-\varepsilon_{0}} \tilde{b}_{+}(x, \xi ; \lambda),
$$

where $\tilde{b}_{+}(x, \xi ; \lambda)$ has the properties $(2.6)-(2.9)$ and $(7.7)$ with the same constant $\mu_{+}$as for $b_{+}(x, \xi ; \lambda)$.

We have thus shown that $Q_{1}(\lambda)$ can be written as a sum of Ps.D.Op.'s: $P_{+}(\lambda)+\langle x\rangle^{-\varepsilon_{0}} P_{+}^{\prime}(\lambda)+P_{N}(\lambda)$, where the symbol of $P_{+}(\lambda)$ is $\overline{b_{+}(x, \xi ; \lambda)}$, the symbol of $P_{+}^{\prime}(\lambda)$ fulfills $(2.6)-(2.9)$ and (7.7) with the same constant $\mu_{+}$as for $b_{+}(x, \xi ; \lambda)$, and the symbol $p_{N}(x, \xi ; \lambda)$ of $P_{N}(\lambda)$ satisfies

$$
\left|D_{x}^{\alpha} D_{\xi}^{\beta} p_{N}(x, \xi ; \lambda)\right| \leqq C_{\alpha \beta}\langle x\rangle^{-|a|-N} .
$$

Next we consider $Q_{2}(\lambda)$. In this case

$$
\left|D_{x}^{\alpha} D_{\xi}^{\beta} D_{y}^{\gamma}(Z(x, \xi, y)-\xi)\right| \leqq C_{\alpha \beta \gamma},
$$

for a constant $C_{a \beta_{1}}$. We rewrite $Q_{2}(\lambda)$ as

$$
\begin{aligned}
& Q_{2}(\lambda) f(x)=\iint e^{i(x-y) \xi} c_{2}(x, \xi, y ; \lambda) f(y) d y d \xi, \\
& c_{2}(x, \xi, y ; \lambda)=a(x, \Psi(x, \xi, y)) \overline{b_{+}(y, \Psi(x, \xi, y) ; \lambda)} \chi_{-}(\hat{x} \cdot \hat{y}) \chi_{\infty}(y) .
\end{aligned}
$$

On the support of $c_{2}(x, \xi, y ; \lambda),|x-y| \geqq C(|x|+|y|)$, since $\hat{x} \cdot \hat{y}<1 / 2$. Taking into account of $\left(1+|x-y|^{2}\right)^{-1}\left(1-\Delta_{\xi}\right) e^{i(x-y) \xi}=e^{i(x-y) \xi}$, we have by integration by parts

$$
\begin{aligned}
& Q_{2}(\lambda) f(x)=\iint e^{i(x-y) \xi} d_{N}(x, \xi, y ; \lambda) f(y) d y d \xi, \\
& \left|D_{x}^{a} D_{\xi}^{\beta} D_{y}^{\gamma} d_{N^{-}}(x, \xi, y ; \lambda)\right| \leqq C_{a \beta{ }^{\prime} N}\langle x\rangle^{-N}\langle y\rangle^{-N}, \text { for any } N \geqq 1 .
\end{aligned}
$$

Hence by Lemma 7.1, $Q_{2}(\lambda)$ turns out to be a Ps.D. Op. with symbol rapidly decreasing in $x . Q_{3}(\lambda)$ can be treated similarly, because its symbol is compactly supported for $y$.

In summary, we have proved the following 
Theorem 7.2. Let $A_{+}, B_{+}(\lambda)$ be as in Definition 2.4. Suppose that $b_{+}(x, \xi ; \lambda)$ satisfies (7.7). Let $P_{+}(\lambda)$ be the Ps. D. Op. with symbol $b_{+}(x, \xi ; \lambda)$. Then for any $N \geqq 1$, there exist Ps. D. Op.'s $P_{+}^{\prime}(\lambda), P_{N}(\lambda)$ having the following properties:

(1) $A_{+} B_{+}(\lambda) *=P_{+}(\lambda)+\langle x\rangle^{-\varepsilon_{0}} P_{+}^{\prime}(\lambda)+P_{N}(\lambda)$.

(2) The symbol of $P_{+}^{\prime}(\lambda)$ fulfills (2.6)-(2.9) and (7.7) with the same constant $\mu_{+}$as for $P_{+}(\lambda)$.

(3) The symbol of $P_{N}(\lambda)=p_{N}(x, \xi ; \lambda)$ satisfies

$$
\left|D_{x}^{\alpha} D_{\xi}^{\beta} p_{N}(x, \xi ; \lambda)\right| \leqq C_{\alpha \beta}\langle x\rangle^{-N-|\alpha|} \text {. }
$$

Here we note that

$$
\langle x\rangle^{-\varepsilon_{0}} P_{+}^{\prime}(\lambda)=\tilde{P}_{+}(\lambda)\langle x\rangle^{-\varepsilon_{0}}+\tilde{P}_{N}(\lambda),
$$

with $\tilde{P}_{+}(\lambda), \tilde{P}_{N}(\lambda)$ having the same properties as $P_{+}^{\prime}(\lambda), P_{N}(\lambda)$, respectively, which follows from the asymptotic expansion of the symbol of the commutator $\left[\langle x\rangle^{-\varepsilon_{0}}, P_{+}^{\prime}(\lambda)\right]$ (see $[1]$, Theorem 2.3). Thus the above theorem is reformulated as follows.

Theorem 7.3. Let $A_{+}, B_{+}(\lambda)$ and $P_{+}(\lambda)$ be as in Theorem 7.2. Then for any $N \geqq 1$, there exist Ps. D. Op.'s $\widetilde{P}_{+}(\lambda), P_{N}(\lambda)$ having the following properties:

(1) $A_{+} B_{+}(\lambda)^{*}=P_{+}(\lambda)+\tilde{P}_{+}(\lambda)\langle x\rangle^{-\varepsilon_{0}}+P_{N}(\lambda)$.

(2) The symbol of $\tilde{P}_{+}(\lambda)$ satisfies $(2.6) \sim(2.9)$ and (7.7) with the same constant $\mu_{+}$as for $P_{+}(\lambda)$.

(3) The symbol of $P_{N}(\lambda)=p_{N}(x, \xi ; \lambda)$ satisfies

$$
\left|D_{x}^{\alpha} D_{\xi}^{\beta} p_{N}(x, \xi ; \lambda)\right| \leqq C_{\alpha \beta}\langle x\rangle^{-N-|\alpha|} \text {. }
$$

Theorem 7.3 one can find a F.I.Op. $B_{+}(\lambda)$ and Ps. D. Op.'s $\tilde{P}_{+}(\lambda)$ and $P_{N}(\lambda)$ such that $P_{+}(\lambda)$ $=A_{+} B_{+}(\lambda)^{*}-\tilde{P}_{+}(\lambda)\langle x\rangle^{-s_{0}}-P_{N}(\lambda)$. Let $\tilde{b}_{+}(x, \xi ; \lambda)$ be the symbol of $\tilde{P}_{+}(\lambda)$. If we take $\tilde{b}_{+}(x, \xi ; \lambda)$ instead of $\overline{b_{+}(x, \xi ; \lambda)}$ in the above procedure, we can obtain the same type of asymptotic expansion for the F.I.Op.

$$
\iint e^{i S(x, \xi, y)} a(x, \xi) \tilde{b}_{+}(y, \xi ; \lambda) f(y) d y d \xi .
$$

Thus $\tilde{P}_{+}(\lambda)$ can be written again as a sum of a F.I.Op. and Ps. D.Pp.'s. Repeating this procedure, we obtain

Theorem 7.4. Let $A_{+}$be as in Definition 2.4. Let $P_{+}(\lambda)$ be the Ps. D. Op. with symbol $p_{+}(x, \xi ; \lambda)$ satisfying $(2.6)-(2.9)$ and $(7.7)$. Then for any $N \geqq 1$ there exist a F. I. Op. $B_{+}(\lambda)$ with symbol $b_{+}(x, \xi ; \lambda)$ satisfying $(2.6) \sim(2.9)$ and $(7.7)$, and $a$ Ps. D. Op. $P_{N}(\lambda)$ having the following properties:

(1) $P_{+}(\lambda)=A_{+} B_{+}(\lambda) *+P_{N}(\lambda)$.

(2) The symbol of $P_{N}(\lambda)=p_{N}(x, \xi ; \lambda)$ satisfies

$$
\left|D_{x}^{\alpha} D_{\xi}^{\beta} p_{N}(x, \xi ; \lambda)\right| \leqq C_{\alpha \beta}\langle x\rangle^{-N-|\alpha|} .
$$




\section{References}

[1] Isozaki, H. and Kitada, H., Micro-local resolvent estimates for two-body Schröinger operators, J. Functional Anal. 57 (1984), 270-300.

[2] —, Modified wave operators with time-independent modifiers, J. Fac. Sci. Tokyo Univ. Section IA, 1985, to appear.

[3] — - Scattering matrices for two-body Schrödinger operators, preprint.

[4] Kumanogo, H., Pseudo Differential Operators, The MIT Press, Cambridge, Massachusets and London, England (1983).

[5] - A calculus of Fourier integral operators on $\boldsymbol{R}^{n}$ and the fundamental solution for an operator of hyperbolic type, Comm in P.D.E., 1 (1976), 1-44. 